\title{
A performance comparison of patient pathways in Nordic capital areas - a pilot study for ischaemic stroke patients
}

\author{
UNTO HÄKKINEN ${ }^{1}$ (D), FANNY GOUDE ${ }^{2}$, TERJE P. HAGEN³ ${ }^{3}$, MARIE KRUSE $^{4}$, \\ TRON MOGER ${ }^{3}$, MIKKO PELTOLA $^{1} \&$ CLAS REHNBERG ${ }^{2}$
}

${ }^{1}$ Centre for Health and Social Economics, Finnish Institute for Health and Welfare, Helsinki, Finland, ${ }^{2}$ Medical Management Centre, Karolinska Institutet, Stockholm, Sweden, ${ }^{3}$ Department of Health Management and Health Economics, University of Oslo, Oslo, Norway, and ${ }^{4}$ Business and Economics, COHERE, Syddansk Universitet, Odense, Denmark

\begin{abstract}
Aims: This article describes and discusses the extension of performance measurement using an episode-based approach so that the measurement includes primary care, and social and long-term-care services. By using data on incident stroke patients from the capital areas of four Nordic countries, this pilot study: (a) extended the disease-based performance analysis to include new indicators that better describe patient care pathways at different levels of care; (b) described and compared the performance of care given in the four areas; (c) evaluated how additional information changed the rankings of performance between the areas; and (d) described the trends in performance in the capital areas. Methods: The construction of data was based on a common protocol that used routinely collected national registers and statistics linked with local municipal registers. We created new variables describing the timing of discharge to home and institutionalisation, as well as describing the use and cost of primary and social hospital services. Risk adjustment was performed with four different sets of confounders. Results: Differences existed in various performance indicators between the four metropolitan areas. The ranking was sensitive to the risk-adjustment method. The study showed that for stroke patients a performance comparison with data that are only from secondary and tertiary care, and without a valid severity measure, is not sufficient for international comparisons. Conclusions: Extending and deepening international performance analysis in order to cover patient pathways, including primary care and social services, is very useful for benchmarking activities when focusing on diseases affecting older people.
\end{abstract}

Keywords: Performance comparison, patient pathways, risk adjustment, ischaemic stroke

\section{Background}

Performance-based governance requires timely and accurate patient data that span the whole care pathway, including health outcomes and costs. Such data are also used to support the redesign and evaluation of new models of health care service delivery and used to contribute to the discovery and evaluation of new treatments. Health care data collected by national registries and other administrative databases, which can be linked with each other at the individual level, are valuable information that enable comparisons of complete care pathways and could generate hypotheses on why health outcomes are better in some countries than in others. Although there is immense potential in these data, they are not routinely used to serve the aims of increasing service system performance. Thus, there is room for promoting their use to support the efficiency objectives of the health care system via benchmarking (i.e. learning from best practices).

Correspondence: Unto Häkkinen, Centre for Health and Social Economics, Finnish Insitute for Health and Welfare, Mannerheimintie 166, P.O. Box 30, Helsinki FI-00271, Finland. E-mail: unto.hakkinen@thl.fi

Date received 16 fuly 2018; reviewed 27 May 2019; accepted 22 fune 2019

(C) Author(s) 2020

Article reuse guidelines: sagepub.com/journals-permissions

DOI: $10.1177 / 1403494819863523$

journals.sagepub.com/home/sjp 
The ability to follow patients as they progress through the health and social care system (from primary health care through specialty visits to hospitalisations, long-term care, home care, hospice care and eventually to death) is essential to health care quality and performance assessment. This type of follow-up allows for a comprehensive view of the health care services provided and the health outcomes generated by those services, and also makes it possible to assess adherence to clinical guidelines, effective treatments, the use of resources and the cost of services.

The data from the national health information systems vary between countries in terms of coverage, coding and terminology, the quality of data, data sharing and linkage possibilities [1]. This is the main challenge of every international performance comparison. In the Nordic countries the registers are well developed, similar in structure and there are possibilities for linkages using personal identity numbers.

A disease-based approach was adopted for evaluating the performance of European health care systems (including those of Finland, Hungary, Italy, the Netherlands, Norway, Scotland and Sweden) as a part of the European Health Care Outcomes, Performance and Efficiency (EuroHOPE) project, using the experiences of the PERFormance, Effectiveness and Cost of Treatment episodes (PERFECT) project [2]. The EuroHOPE project developed methods [3-5] and indicators [6] for international register-based health care performance measurement and comparison, giving proposals concerning the data content of national registers in order to improve national- and international-level continuous monitoring and to implement European-wide health care benchmarking. More recently, national-, regional- and hospital-level indicators have been calculated for Finland, Denmark, Hungary, Italy, Norway and Sweden (http://www. eurohope.info). In a recent pilot study, the databases have been extended to include primary health care and social services using data from the capital areas of Denmark, Finland, Norway and Sweden. The pilot study includes acute myocardial infarction, acute coronary syndrome, stroke and hip fracture patients [6]. This paper describes the methods, data collection and experience of the pilot study using stroke patients as an example. We concentrate on stroke patients because they are typically old and frail and consume services along a pathway that includes hospital care, primary care, rehabilitation and long-term-care services.

\section{Aims}

Using linkable patient-level data on incident stroke patients, the pilot study will (a) extend disease-based performance analysis to include new indicators that better describe patient care pathways at different levels of care; (b) describe and compare the performance of care given in the four metropolitan areas; (c) evaluate how additional data (primary and social services, better risk adjustment and new outcome measures) change the rankings of performance between the areas and discuss their usefulness in regard to better understanding the reasons behind performance differences; and (d) describe the trends in performance between the metropolitan areas.

\section{Data and methods}

This study used linkable, patient-level data on incident stroke patients from the capital areas of Copenhagen (population: 569,557 in 2014), the Helsinki area (the municipalities of Helsinki and Espoo - hereafter, Helsinki: population: 886,260), Oslo (population: 641,550) and Stockholm (the county; population: $2,198,044)$. The construction of the data was based on a common protocol using routinely collected national registers and statistics on hospital discharges, the use of prescribed medication and causes of death $[3,4]$. We defined an episode of care as referring to the entire treatment pattern from the beginning (i.e. the time of diagnosis) of the stroke to the end of the treatment across organisational boundaries within a specific time frame. Thus, the protocols for an episode include the definitions of start and end dates (the follow-up time) as well as inclusion and exclusion criteria, which are used when constructing comparison data.

For each patient, all continuous hospital treatment (from the first hospital episode) starting from the first stroke (cerebral infarction [ICD-10 code I63], intracerebral haemorrhage [ICD-10 code I61], subarachnoid haemorrhage [ICD-10 code I60], or an ill-defined stroke [ICD-10 code I64]) admission (index admission) in every year was constructed by combining all consecutive hospital stays for each patient. The consecutive hospital stays did not need to be in the same hospital; in other words, hospital transfers were taken into account when constructing the first hospital episode. In the case where a patient had different stroke subtypes or ill-defined stroke diagnoses during the first hospital episode, the most 'severe' diagnosis was chosen to characterise the condition of the patient. For this purpose, the following hierarchy of stroke subtypes was applied: subarachnoid haemorrhage, intracerebral haemorrhage, cerebral infarction and an ill-defined stroke. The construction of patient cohorts is described in more detail elsewhere [8].

Each partner was individually responsible for producing its own local EuroHOPE comparison database using the principles stated in the protocol. In order to increase the comparability of the data, we 
only included cerebral infarction (an ischaemic stroke) based on the above-mentioned hierarchy using two exclusions. First, we excluded all patients with a stroke admission (a hospital discharge record with a stroke diagnosis as the main diagnosis) during the previous 365 days before the index admission. Second, we excluded patients under 18 years of age, foreigners and patients with an incomplete personal identity number.

For this pilot study, we extended the approach to primary health care and long-term-care services by collecting data on these services from local municipal registers. We constructed a comparative database that allows for performance analysis, research and the use of indicators at the local (capital area) level (online Supplemental Material 1). Our approach required patient-level data covering the whole population in the selected areas and the possibility to deterministically link individuals' records in the different national and local registers used.

For this study we created new variables that also covered the use of primary and social services. To track patients' movements along the care pathway, we constructed a STATE variable that describes in which place the patient was and what state he or she was in each day 365 days before and 365 days following the index day [9]. The STATE variable is based on the idea that a patient can only be at one specific place each day and conveys information about (a) the patient's fundamental state (dead, alive at home or alive at an institution); (b) the type of care (hospital, rehabilitation, nursing home, home nursing or other types of care); (c) the main diagnosis and intensity of the treatment (i.e. acute care or non-acute care); and (d) types of outpatient visits.

In addition, we collected new variables describing the total number of various visits within 90 and 365 days before and after the index day. The visit categories were as follows:

a) Day surgery

b) Outpatient visits to hospital

c) Outpatient physician consultation with a private or public specialist outside a hospital

d) GP visits

e) Consultations with local emergency centre patients

f) Home visits by a doctor

g) Home care (help) visits.

\section{Performance measures}

Based on the STATE variable and jointly agreed definitions, we used 18 performance indicators that can be divided into five groups (see Tables I and II).
Variables describing mortality included 30-day, 90-day and 1-year mortality.

Variables describing emplacement included the share of patients permanently discharged to home within 90 days from the index day, the share of patients permanently discharged to home without help within 90 days, the share of patients institutionalised within 90 days and the share of patients institutionalised within 1 year from the index day. Here, permanently means that a patient must be at least 2 weeks continuously at home after discharge, that is, without being transferred to any inpatient care (including respite care) within that period. Institutionalisation was defined as the patient being in inpatient care every day during the follow-up time (90 days or 1 year).

Variables describing length of stay (LOS) were defined in three ways: those for an acute hospital episode (including transfers between acute hospitals), those for an acute care and rehabilitation episode, and those for the first institutional episode that also includes, in addition to the acute hospital care and rehabilitation, long-term-care institutions like nursing homes during the first 90 days after the index day if a patient is transferred to such institutions immediately after acute care and rehabilitation admissions.

Variables describing service use included the number of inpatient days, the number of GP visits, the number of other health care visits and the number of home care visits, all calculated for a 1-year period after the index day. Home help included both practical assistance and home nursing because these services cannot be distinguished in the Finnish data. There were differences in the content of non-institutional register data. Home help was not measured using the same units (being measured by visits in Helsinki and by hours in both Oslo and in Copenhagen). Our estimates were based on the assumption that 1 hour equals two visits, which is based on a crude estimation by municipal civil servants in Helsinki.

Variables describing costs included the cost of the first hospital episode using the three definitions described above and 1-year costs including, in addition to institutional care, the use of other services (GP visits, other visits and home help services). Costs in euros (EUR) were measured by using the Finnish standard cost of specific cost items [7,10], which were deflated to 2014 price levels. In acute hospital care, costs were estimated using the average cost per day of stroke patients treated at Helsinki University Hospital (EUR 822).

\section{Missing data}

We only had full data covering the cohorts from the years 2009-2014 from Helsinki. For Copenhagen we 


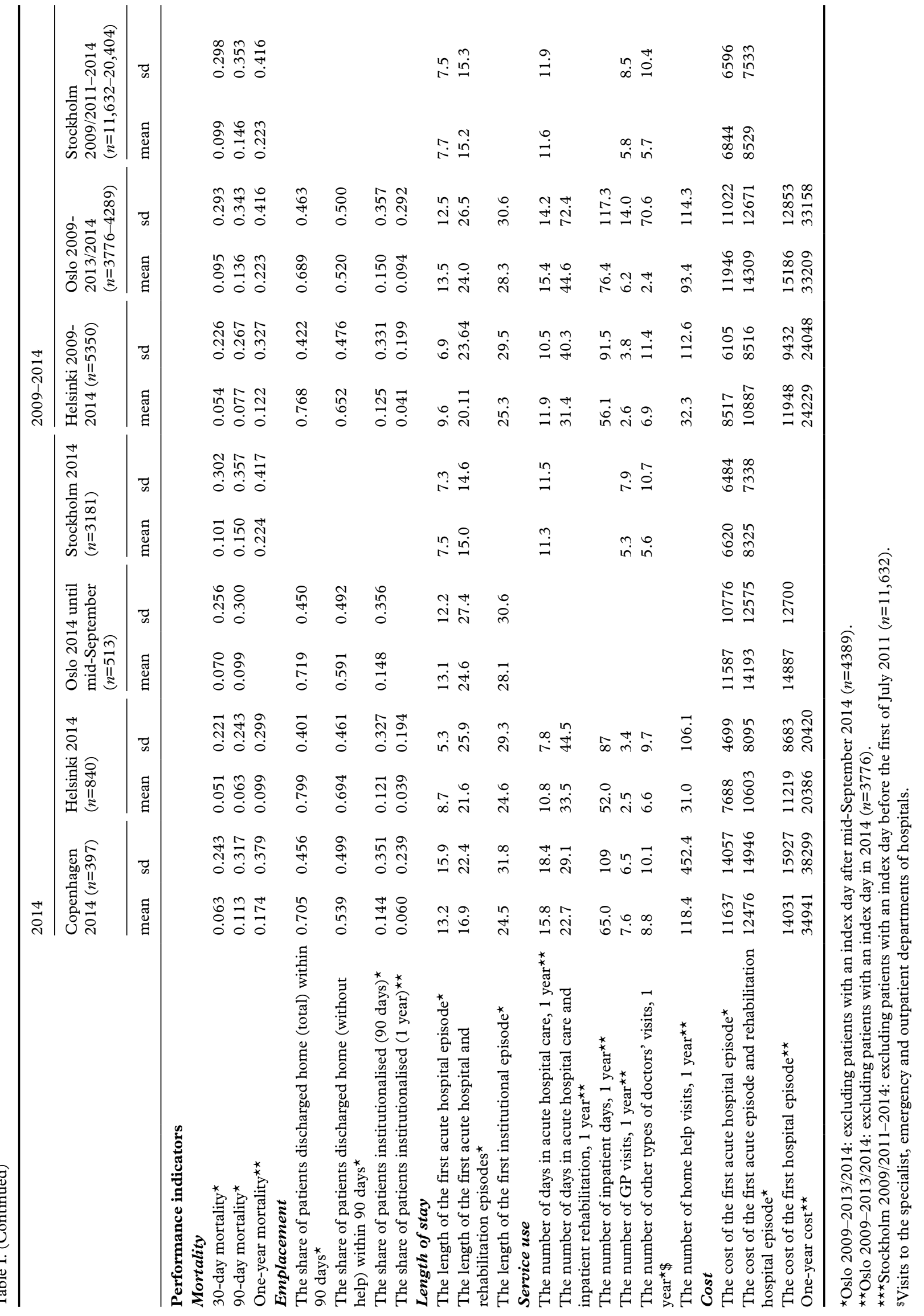




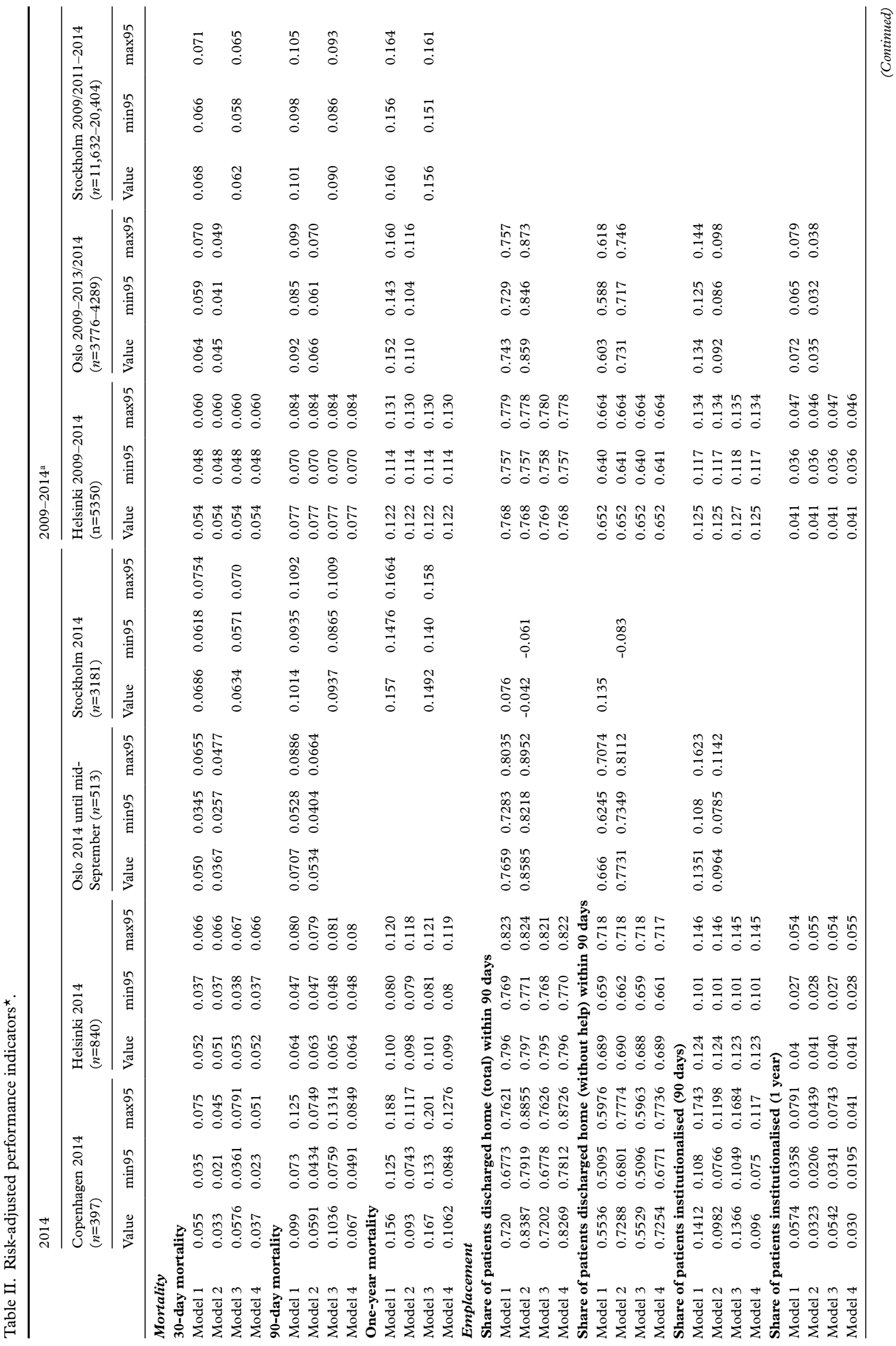




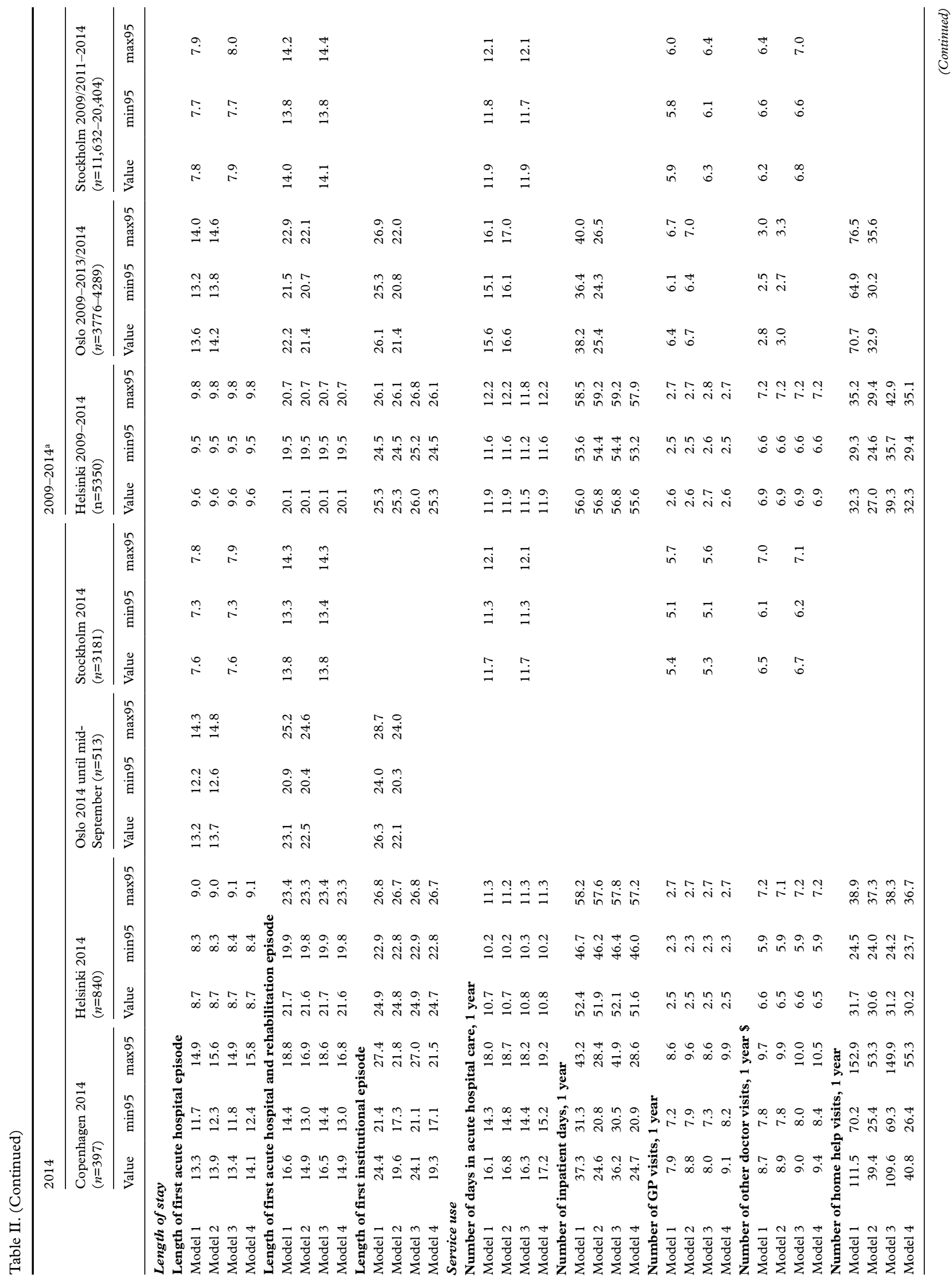




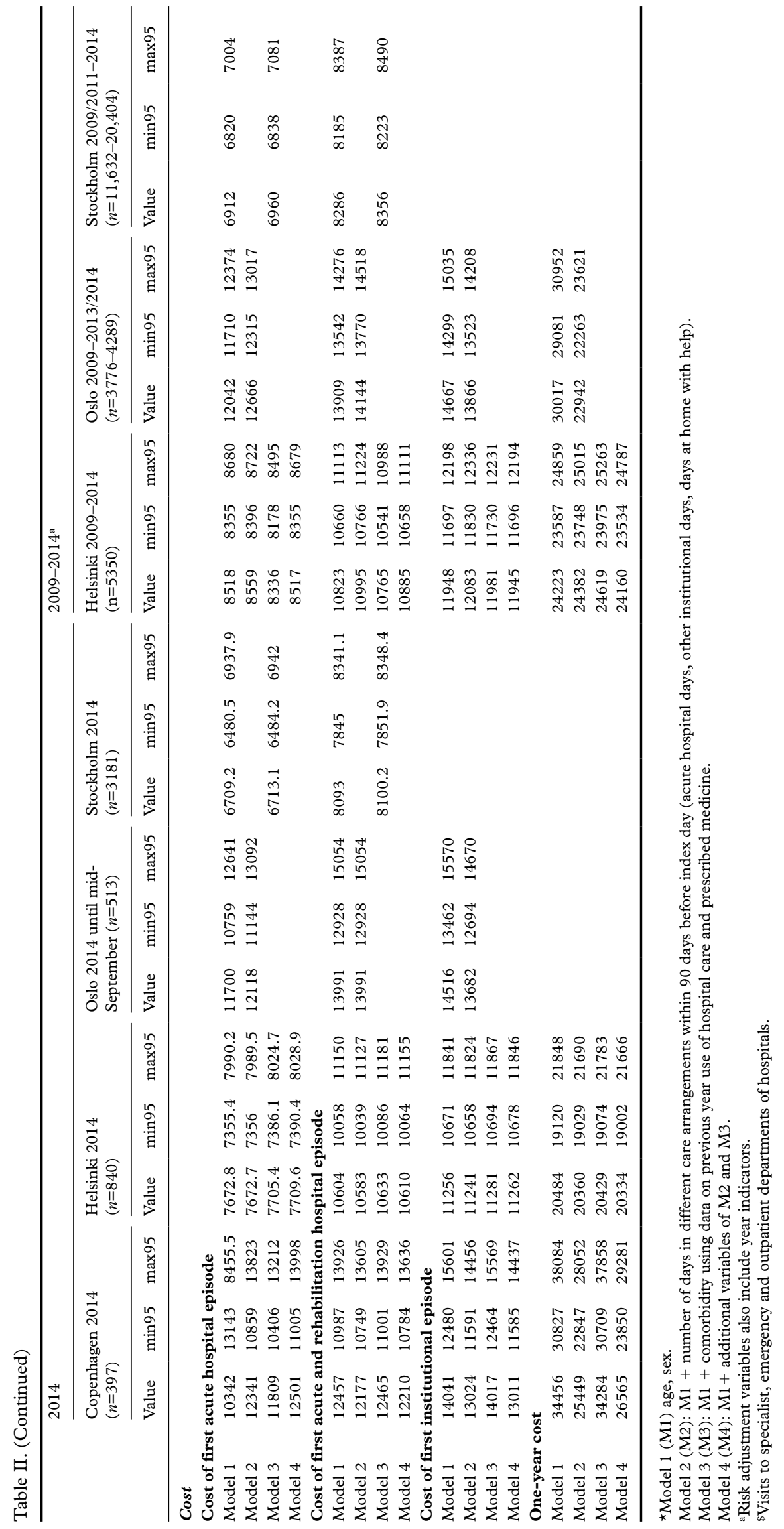


only had full data for the 2014 cohort. For Stockholm, the data covered the cohorts for 2009-2014 but did not include information on institutional long-term care or home help services (except for home visits by nurses and doctors). Data on prescribed medicines from Stockholm was only available from July 2010. Thus, the indicators based on prescribed medicines could only be calculated for patients with an index day after July 2011. For Oslo it was not possible to obtain data on prescribed medicines. Furthermore, we could only follow patients to the end of the year 2014. Thus, we could not calculate the indicators that required a 365-day follow-up for patients treated in 2014. For the variables based on a shorter followup, we excluded patients with an index day after midSeptember of 2014. Online Supplemental Material 2 describes the main performance indicators and their availability by the metropolitan areas.

Because of the missing information, we report the main results (see Tables I and II) using two sets of comparisons. First, we compare all four areas using data from the year 2014. Second, we compare Helsinki, Oslo and Stockholm using data from all 6 years.

\section{Risk adjustment}

Patient-associated factors must be accounted for when comparing the areas. We have endeavoured to ensure meaningful comparisons using three steps. First, we defined the disease groups so that they are as comparable and homogeneous as possible. Second, we gathered information on risk factors from the patients' medical history and patient use of services before the onset of the disease (based on the STATE variable). Third, we applied statistical models to adjust the indicators and calculated their $95 \%$ confidence intervals.

Age and sex are the most commonly used variables in risk adjustment. In addition to these, we used two additional sets of variables: the number of days a patient had been in different service settings before the index day and comorbidities. Using the STATE variable, we calculated the number of days the patient had been in acute hospital care, other institutional care and receiving home help during the 90 days before the index day.

Comorbidities were assessed using patients' medical records for the previous year from two data sources: (a) records based on the primary or secondary diagnoses recorded during hospital admissions within 365 days prior to the index admission and (b) records based on the purchase of medications that can be linked to particular diagnoses [4]. We calculated the comorbidity for 12 diseases: hypertension, coronary artery disease, atrial fibrillation, cardiac insufficiency, diabetes mellitus, atherosclerosis, cancer, COPD and asthma, dementia, depression, Parkinson's disease and mental disorders.

Likewise, the availability of data for various performance measures and risk-adjustment variables varied between the capital areas. Thus, for each performance indicator we performed risk adjustment with four different sets of confounders (see online Supplemental Material 3):

- Model 1 (M1): age, sex

- Model 2 (M2): M1 + the number of days in different care arrangements within 90 days before the index day (acute hospital days, other institutional days, days at home with help)

- Model 3 (M3): M1 + comorbidity using data on the previous year's use of hospital care and prescribed medicines

- Model 4 (M4): M1 + the additional variables of $\mathrm{M} 2$ and M3.

M2 and M4 hence account for the use of health services prior to admission. The outcome of these models can therefore be interpreted as the development in service utilisation, whereas M3 describes the level of health.

For all performance indicators, a modelling strategy for risk adjustment was adopted: logistic regression for dichotomous responses (e.g. mortality), generalised linear modelling for continuous variables (e.g. costs' gamma distribution with a log link) and negative binomial modelling for count variables (e.g. the LOS). Ideally, the individual-level data from all participating areas would be pooled before estimating the risk-adjustment models. However, at this stage we did not have permission from all the partners to pool the individual-level data. Thus, we applied a standard approach for indirect standardisation in which the parameter estimates for the confounding factors were first estimated for each performance indicator using the data from Helsinki from the years 2009-2014. Then, the coefficients of each model were made available to all partners who then calculated individuallevel predicted values for the indicators. The predicted values were then summed up for an area by year. The ratio of the observed value and the expected value of the dependent variable in the comparable unit was multiplied by the average value of the indicator in Helsinki data in order to constitute the risk-adjusted indicator. Thus the adjusted estimates correspond to assuming that the effects of all risk adjusters are the same as in Helsinki.

The calculation of the risk-adjusted indicators using data for all years (see Table II; 2009-2014) was 


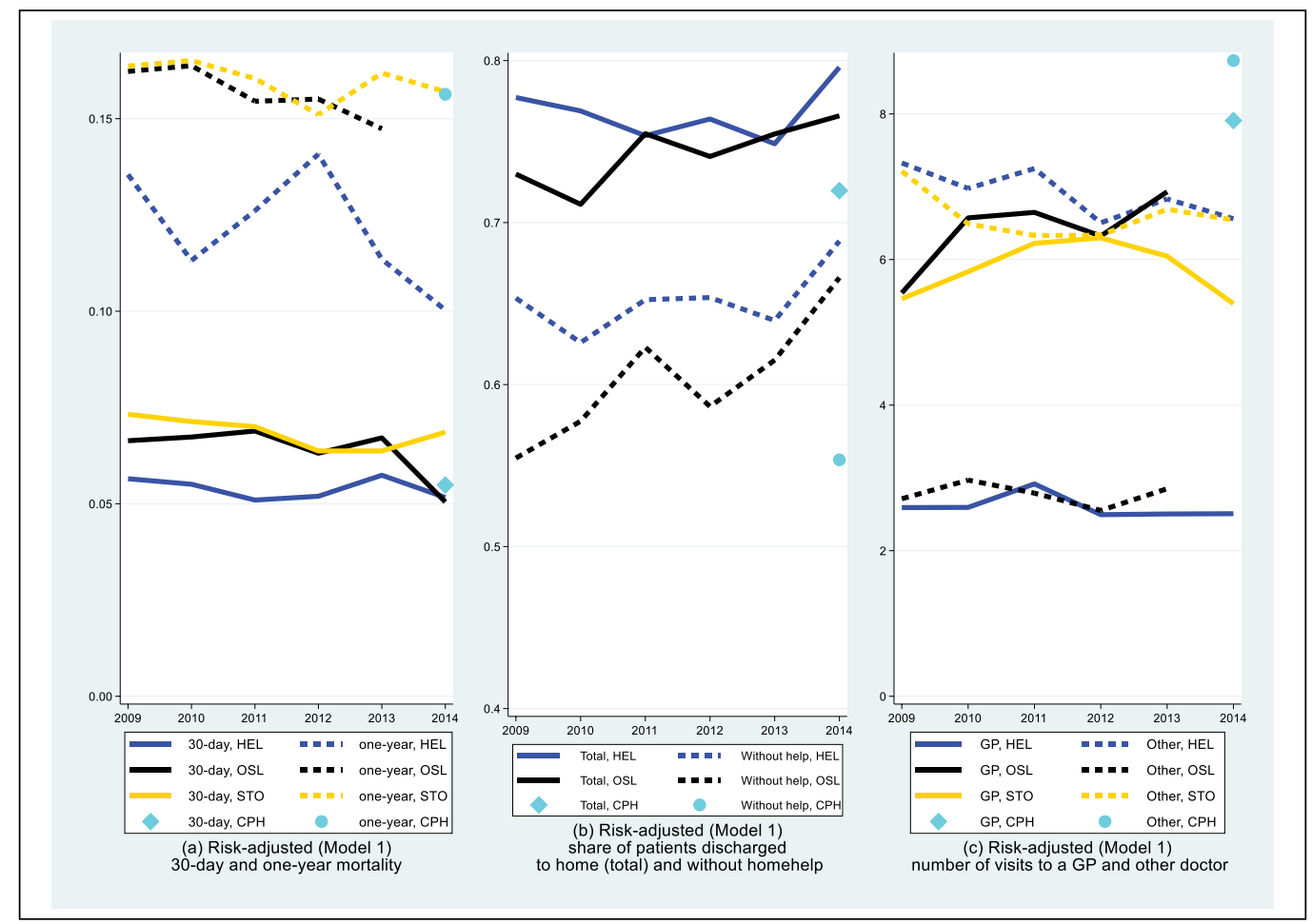

Figure 1. The risk-adjusted trends of mortality (Figure 1(a)), the share of patients discharged to home (Figure 1(b)) and the use of doctors' services (Figure 1(c)) in Helsinki (HEL), Oslo (OSL), Stockholm (STO) and Copenhagen (CPH) 2009-2014 and after the onset of a stroke.

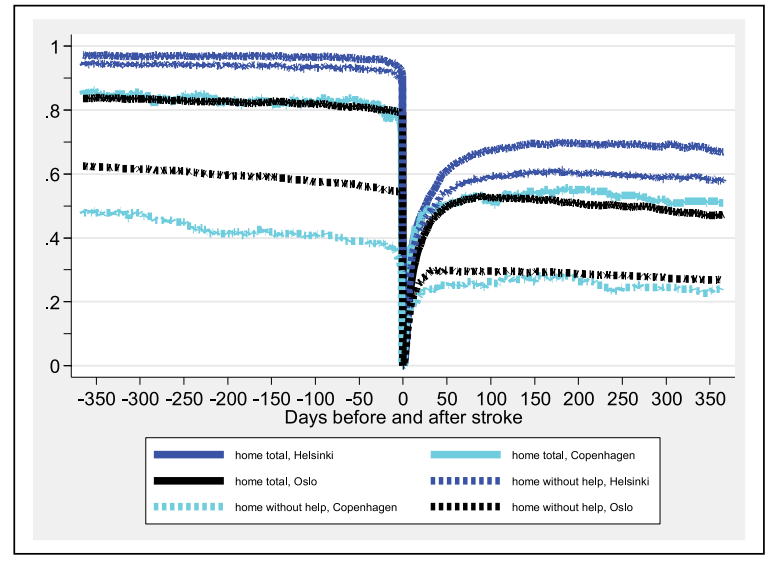

Figure 2. The daily shares of patients at home in total and those at home without help before and after onset of a stroke; the patients were over 74 years of age.

based on models that also included year dummies, whereas indicators from the year 2014 (Table II; 2014) and the figures (describing annual trends; see Figures 1 and 3) of risk-adjusted indicators did not include the year dummies. The age- and sex-standardised incidence figures for ischaemic strokes were carried out via indirect standardisation using the age and sex reference values for Helsinki for the years 2009-2014 and this was used as the basic population.

\section{Results}

\section{Patient structure}

The age- and sex-standardised number of incident ischaemic stroke patients per 10,000 people Helsinki was 10.4 (varying over the years between 9.2 and 11.3), in Stockholm it was 15.6 (13.7-16.7), in Copenhagen it was 9.0 (2014) and in Oslo it was 14.0 (13.2-14.6). The patients were older in Oslo and Stockholm compared with Helsinki and Copenhagen (see Table I). In addition, in 2014 the share of patients who was in hospital or long-term care during 90 days before the index day were higher in Oslo $(9 \%)$ and Copenhagen $(7 \%)$ compared with Helsinki (1\%).

\section{Mortality}

In 2014 unadjusted 30-day mortality rates were higher in Stockholm than in Helsinki, Oslo and Copenhagen (see Table I). Also, age- and sexstandardised 30-day mortality (M1) was highest in Stockholm but the differences between the areas were not statistically significant. However, the figure (for M1) for the whole study period shows statistically significantly higher values for Stockholm compared with Helsinki. When adjusting for the previous 


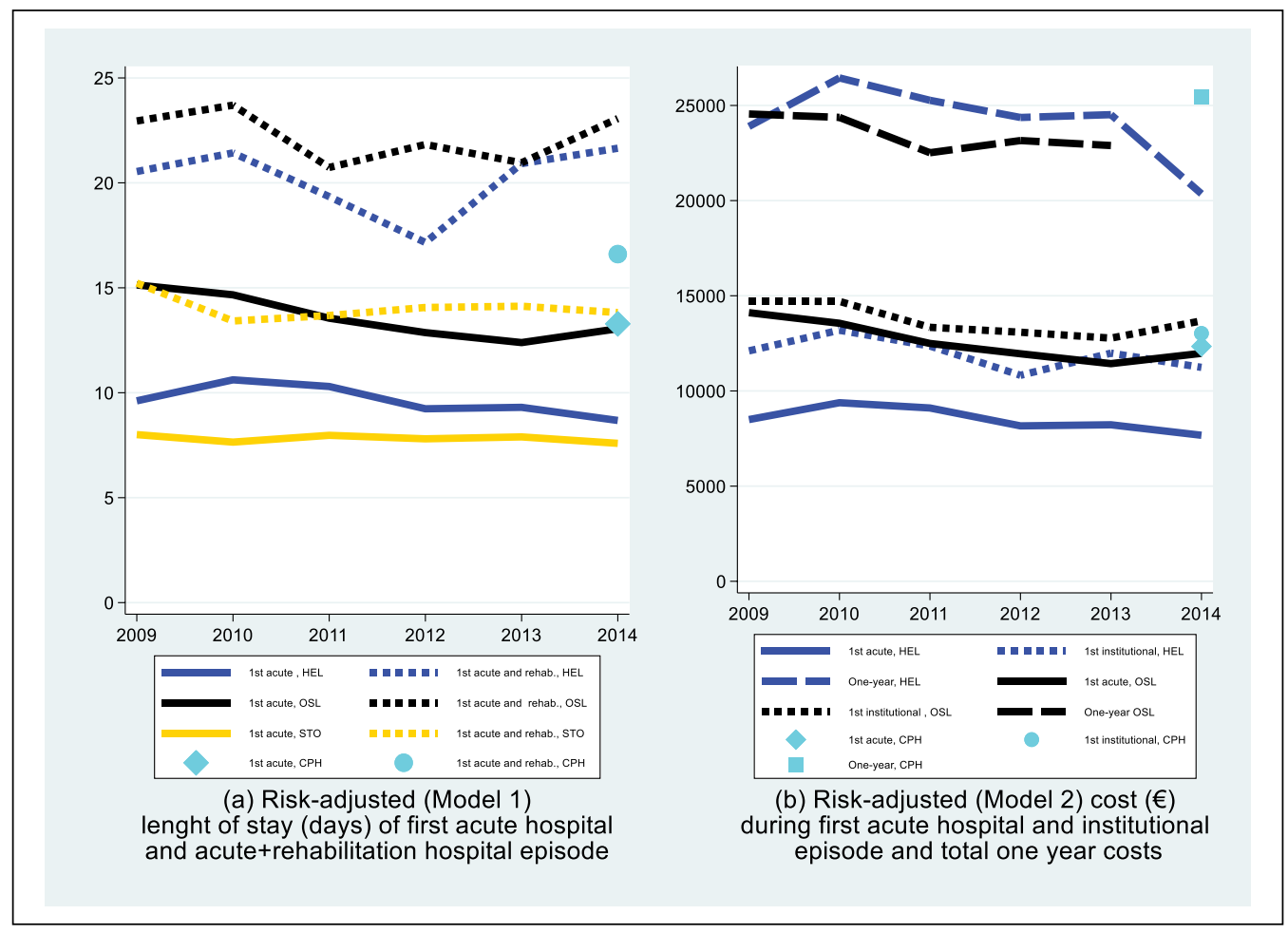

Figure 3. The risk-adjusted length of stay of the first hospital stay (Figure 3(a)) and the cost of the first hospital episode and total 1-year costs (Figure 3(b)) in Helsinki (HEL), Oslo (OSL), Stockholm (STO) and Copenhagen (CPH) during 2009-2014 and after the onset of a stroke.

use of services before the index day (M2), the three mortality indicators were even somewhat lower in Oslo (2014 and whole study period) and Copenhagen (2014) than in Helsinki (see Table II). All the available 90-day and 1-year mortality indicators were higher in Stockholm compared with Helsinki. The age- and sex-standardised (M1) mortality figures were rather stable during the study period while 1 -year mortality only decreased between the years 2009 and 2010 and between 2012 and 2014 (Figure 1 (a)) in Helsinki. In addition, in 2014 30-day mortality in Oslo decreased to the level of Helsinki and Copenhagen.

\section{Emplacement}

Using a diagram constructed from the STATE variables, we were able to describe the unadjusted share of patients at home before and after the index day. Figure 2 describes the daily share of patients over 74 years of age that were at home during a 1 -year period before and after the index day. Before the onset of a stroke, the share was about 12 percentage points higher in Helsinki than in Oslo and Copenhagen. In Helsinki patients also returned home more quickly.

The picture is quite different when we consider the patients who were at home without home help services. The share of patients at home without home help before a stroke was clearly higher in Helsinki than in Oslo and Copenhagen. The use of home help services was more frequent in the two cities during 90 days prior to the index date (see Table I). For example, 1 week before the index day, $45 \%$ of patients (over 74 years of age) in Copenhagen and $25 \%$ of patients in Oslo received home help services compared with only $3 \%$ in Helsinki.

In Helsinki, the age- and sex-standardised share of patients discharged to home within 90 days (M1) in 2014 was almost eight percentage points higher than in Copenhagen and about three percentage points higher than in Oslo. The corresponding figure for discharges to home without help was even higher (13 percentage points) in Helsinki compared with Copenhagen. However, when the differences in the utilisation of services before the onset of a stroke are taken into account (M2), the share of patients permanently discharged to home was about six percentage points higher in Oslo compared with Helsinki for all home discharges and about eight percentage points higher for discharges without home help (see Table II). The adjusted shares (M2 and M4) in Copenhagen were also somewhat higher than in Helsinki, but the differences were not statistically significant, though during the study period Oslo reached 
the level of Helsinki in age- and sex-standardised (M1) shares (see Figure 1(b)). Also, the risk-adjusted shares of patients institutionalised during 90 days and 1 year after stroke were sensitive to the riskadjusting model (see Table II).

\section{The use and cost of services}

The use and structure of outpatient doctor services after 1 year from the onset of a stroke varied between the areas. These figures were not sensitive to the riskadjusted model. The number of GP visits and other doctor visits (including visits to the specialist, emergency and outpatient departments of hospitals) was highest in Copenhagen. Also, in Stockholm the number of both types of visits was relatively high, whereas in Oslo GP visits were the dominant type and in Helsinki other visits were the dominant type. During the study period, the use of GP services (M1) in Oslo increased (see Figure 1(c)). In 2014 home help services were provided more often to patients in Copenhagen than to those in Helsinki. Also, the figures adjusted for the previous use of services (M2) were slightly higher in Oslo (whole study period) and Copenhagen (2014) than in Helsinki (see Table II).

The LOS of inpatient care during the first acute episode was shortest in Stockholm. When inpatient rehabilitation was added to first acute episode, the LOSs were lowest in Stockholm and Copenhagen (2014). In Oslo the LOS during the first acute episode was longer than in Helsinki, but when rehabilitation admissions were also taken into account, the difference between Helsinki and Oslo diminished (see Table II). In Helsinki the use of care given at health centres during the first hospital episode increased after 2012. Except for this change, the annual changes in the age- and sex-standardised (M1) LOS during the first acute hospital episode and the following inpatient rehabilitation period were minimal in the three areas (see Figure 3(a)).

The risk-adjusted (M1 and M2) number of all inpatient days during the first institutional episode was about the same in the three areas in 2014 but the number of inpatient days during 1 year was lower in Oslo (for all years) and Copenhagen (2014) than in Helsinki (see Table II). But since stroke patients in Oslo and Copenhagen used more expensive acute hospital care, all the adjusted cost figures for the care during the first hospital episode were somewhat higher in Oslo and Copenhagen compared with Helsinki. Also, the 1-year costs were sensitive to the risk-adjustment method. In 2014 age- and sex-standardised (M1) 1-year costs were about EUR 14,000 higher per patient in Copenhagen than in the Helsinki area, whereas during the whole study period the costs were EUR 6,000 lower in the Helsinki area than in Oslo. The main reason for the higher cost in Copenhagen was related to the greater use of acute hospital care, as well as of ambulatory and home help services. When M2 was used for risk adjustment, the difference between Copenhagen and Helsinki was reduced to EUR 5,000 and the difference between Helsinki and Oslo was no longer statistically significant (see Table II). During the years 2009-2013 the adjusted (M2) total 1-year costs were about EUR 25,000 in Helsinki and about the same level as in Copenhagen in 2014 (see Figure 3(b)). In 2014 they decreased in Helsinki by EUR 5,000 EUR per patient.

\section{Discussion}

As health care systems may have a different role for primary, secondary, tertiary and social care services, the impact these sectors have on patient outcomes may also vary.

To the best of our knowledge, this was the first international comparison where register data from primary- and long-term-care services were linked with hospital discharge data and mortality registers. The study shows that for patients with mixed care pathways across primary, secondary, tertiary and social services, a performance comparison with data that are only from secondary care and without a valid severity measure of a disease is not always sufficient for international comparisons. Our approach gave us an opportunity to evaluate outcome measures other than mere survival, such as measures describing the increase in quick discharges to home (with and without help) and the reduction in institutionalisation, which have been important policy aims in all the Nordic countries. Such measures can be used as the proxy variables of outcomes if we can assume that the measures reflect the functional status of patients to some extent.

The approach presented here is an important extension in the field of health system performance analysis. With individual level routinely collected data, it is possible to considerably deepen the analysis and enrich the set of outcome and process indicators available for system performance comparison.

Our study highlights several challenges that need to be taken in account in international performance comparisons. The results underline the importance of adequate risk adjustment in order to reduce the bias associated with patient selection. The ranking of various performance indicators between the four areas was sensitive to the risk-adjustment method used. In particular, the previous use of different services (inpatient care, home help) as covariates in the adjusted models changed the rankings between the 
areas in terms of the measures describing the use of inpatient care and costs during a longer follow-up period. By including them, we assumed that the previous use of services was closely related to the functional ability of patients. However, we cannot confirm that the functional status of people living at home with or without help is the same between the regions because it may to some extent reflect differences in policy priorities. On the other hand, the inclusion of these measures in risk adjustments can be justified if they reflect changes in the use of services because of the onset of a disease, and these changes can be affected by treatments or interventions.

In addition, risk adjustment based on age and sex, and even comorbidities based on the medical history of patients, may not be enough for a reliable performance comparison of diseases affecting older persons. Information on the severity of a stroke (see e.g. Fonarow et al. [11]) is not routinely available from administrative registers. Our study indicates that functional ability (such as the measures of activities undertaken in daily living) before the onset of disease may also be an important predictor of performance that should be taken into account in the risk adjustment.

In this pilot study it was not possible to pool data from different countries, and risk adjustment was done using coefficients calculated from Helsinki. In addition, the coverage and years of availability of the data varied between areas. An analysis will be made more reliable and carried out more easily if the data can be pooled and if other information, such as socioeconomic variables, can be included, enabling possibilities to, for example, evaluate the effect of a reform made in one area using other areas as control groups [12]. Current technology (e.g. TSD - Services for sensitive data at the University of Oslo) allows a pooled analysis to be made in a secure data portal and secure facilities in order to protect data privacy (https://www. uio.no/english/services/it/research/sensitive-data/).

One special challenge is to increase the comparability of register data describing non-institutional services. For example, home help was not measured using the same units (it was measured by visits in Helsinki and by hours in Oslo and in Copenhagen). Our estimates were based on the assumption that 1 hour equals two visits. In addition, the hospital costs in Stockholm might be underestimated, as a short LOS would involve higher cost/bed-day as treatment is more resource intensive.

Our approach to measuring costs by means of resource use has both strengths and weaknesses. The main advantage is that we can avoid concerns about differences in cost-accounting systems and prices between the countries and producers. It is also an easy way to combine different services. The main weakness of this approach is that it does not take into account the differences in the unit prices of resource items across countries. For example, the costs of inpatient days, procedures or visits may vary owing to differences in inputs (personnel, working time, etc.) and we assumed that the relative costs of these cost items are the same in all countries. Thus, we compared resource use using the Finnish estimates for the average unit cost of services, not actual spending, and our cost measure does not necessarily reflect actual expenditure differences.

Despite these shortcomings, our results indicate some differences between the capital areas reflecting important policy issues. First, the considerable differences in age- and sex-standardised incidence figures, as well as in the age structure of patients, may reflect differences in the role of acute care in treatment patterns as well as more aggressive and resource-intensive treatment in Norway and Sweden. In Oslo, for example, the average age of the total population is much younger compared with Helsinki and Copenhagen [7], while the average age of the Norwegian patients was much older. Alternatively, the age of onset may just be higher in Oslo and Stockholm due to a better lifestyle or better managed stroke risk factors. Second, in Oslo the ambulatory services mainly comprised services from GPs, while in Copenhagen and Stockholm ambulatory services included both those of GPs and hospital outpatient services, and in Helsinki they merely included hospital outpatient services. In addition, the use of home help services both before and after the onset of a stroke was considerably higher in Copenhagen and Oslo compared with Helsinki. This may indicate more developed primary and home help services in Norway and Denmark when compared with Finland, as can be assumed based on descriptive information on the primary care systems [13]. However, these differences were not associated with better outcomes in Copenhagen and Oslo compared with Helsinki.

\section{Conclusions}

The extending and deepening of international performance analysis in order to cover patient pathways including primary care and social services is very useful for benchmarking (i.e. learning from best practices) activities focusing on the diseases affecting older people. It increases our understanding of the challenges in international performance measurement. General policy pressure in all Nordic countries to treat more patients at home can be captured using the new indicators presented here. The Nordic performance analysis can be developed by harmonising the cost 
information and content of registers describing the use of services outside hospitals, as well as those gathering routine information on measures of activities involved in daily living for the elderly population. The quality of Nordic performance comparisons will improve considerably if individual-level data can be pooled through a secure data portal and facilities.

\section{Declaration of conflicting interests}

The authors declare that there is no conflict of interest.

\section{Funding}

The data gathering and analyses were funded by the Health Programme of the European Union (grant number 664691 / BRIDGE Health) and the Norwegian Research Council (grant number 229092 / "Comparative effectiveness analyses of coordinated care initiatives in three Nordic countries"). Unto Häkkinen acknowledges financial support from the Yrjö Jahnsson Foundation and the Finnish Society for Health Economics.

\section{ORCID iD}

Unto Häkkinen (iD https://orcid.org/0000-0002-6811 $-0009$

\section{Supplemental material}

Supplemental material for this article is available online.

\section{References}

[1] OECD. Health Data Governance: Privacy, Monitoring and Research, OECD Health Policy Studies. Paris: OECD Publishing, 2015.
[2] Häkkinen U. The PERFECT-project: measuring performance of healthcare episodes. Ann Med 2011;43(Suppl. 1):S1-3

[3] Häkkinen U, Iversen T, Peltola $M$, et al. Health care performance comparison using a disease-based approach: The EuroHOPE project. Health Policy 2013;112:100-9.

[4] Moger TA and Peltola M. Risk adjustment of health-care performance measures in a multinational register-based study: A pragmatic approach to a complicated topic. $S A G E$ Open Med 2014;2:2.

[5] Iversen T, Aas E, Rosenqvist G, et al. Comparative analysis of treatment costs in EuroHOPE. Health Econ 2015;22(Suppl. 2):5-22.

[6] Malmivaara A, Meretoja A, Peltola $M$, et al. Comparing ischaemic stroke in six European countries: The EuroHOPE register study. Eur F Neurol 2015;22:284-91, e25-6.

[7] Häkkinen U, Engel-Andreasen C, Goude F, et al. Performance comparison of patient pathways in Nordic capital areas: A pilot study. THL, Discussion Paper 22/2018.

[8] EuroHOPE. Building register-based performance indicators for stroke using individual-level administrative health care data, http://www.eurohope.info/ (2016, accessed 27 August 2016).

[9] Sund R and Häkkinen U. Using registry data to compare health care efficiency. In: Cylus J, Papanicolas I and Smith P (eds) Health System Efficiency: How to Make Measurement Matter for Policy and Management. European Observatory on Health Systems and Policies Policy Series 46. 2016, pp.5374.

[10] Kapiainen S, Väisänen A and Haula T. Terveyden- ja sosiaalihuollon yksikkökustannukset Suomessa vuonna 2011. THL raportti 3/2014.

[11] Fonarow GC, Alberts MJ, Broderick JP, et al. Stroke outcomes measures must be appropriately risk adjusted to ensure quality care of patients. A Presidential Advisory from the American Heart Association/American Stroke Association. Stroke 2014;45:1589-601.

[12] Häkkinen U, Hagen TP and Moger TA. Performance comparison of hip fracture pathways in two metropolitan areas: Associations with level and change of integration. Nord $\mathcal{F}$ Health Econ 2017;6:80-98.

[13] Häkkinen U and Jonsson PM. Harnessing diversity of provision. In: Magnussen J, Vrangbæ K and Saltman R. B. (eds) Nordic Health Care Systems: Recent Reforms and Current Policy Challenges. Open University Press, 2009, pp.198-213. 